\title{
Identification, prevalence, and treatment of painful diabetic neuropathy in patients from a rural area in South Carolina
}

This article was published in the following Dove Press journal:

Journal of Pain Research

7 April 2017

Number of times this article has been viewed

\author{
Jimmy Pruitt III' \\ Carolina Moracho- \\ Vilrriales ${ }^{1,2}$ \\ Tiffaney Threatt ${ }^{3}$ \\ Sarah Wagner ${ }^{3}$ \\ Jun Wu'
}

\section{E Alfonso}

Romero-Sandoval'

'Department of Pharmaceutical and Administrative Sciences, Presbyterian College School of Pharmacy, Clinton, SC, USA; '2Department of Biochemistry and Biotechnology, University of Alcalá de Henares, Madrid, Spain; ${ }^{3}$ Department of Pharmacy Practice, Presbyterian College School of Pharmacy, Clinton, SC, USA
Correspondence: E Alfonso Romero-Sandoval

Department of Pharmaceutical and Administrative Sciences, Presbyterian College School of Pharmacy, 307 North Broad Street, Clinton, SC 29325, USA

Tel + I 8649383833

Fax +l 8649383903

Email asandoval@presby.edu
Abstract: Diabetic peripheral neuropathy (DPN) represents significant burdens to many patients and the public health-care system. Patients with diabetes in rural areas have higher risk of developing complications and having less access to proper treatment. We studied a rural population of patients with diabetes who attended a pharmacist-led free clinic for a diabetic education program. Our objectives were to 1) determine the prevalence of DPN and painful diabetic neuropathy (p-DN) in patients with type 2 diabetes; 2 ) assess the proportion of patients with DPN and p-DN left undocumented upon physician referral to a pharmacist-led free clinic; and 3) determine the appropriateness of pain medication regimen. We performed a retrospective analysis of clinical records of patients from the Presbyterian College School of Pharmacy (PCSP) Wellness Center located in Clinton, SC. Diagnoses of DPN and/or p-DN were obtained from referral notes in the clinical records and compared with results from foot examinations performed in the free clinic and clinical features. Medication regimens were also obtained and compared using American Academy of Neurology (AAN) treatment guidelines. Within our study population $(\mathrm{n}=111)$, the prevalence of DPN was $62.2 \%$ (national average of $28 \%-45 \%$ ) and that of $\mathrm{p}-\mathrm{DN}$ was $23.4 \%$ (national average of $11 \%-24 \%$ ). In $\mathrm{p}-\mathrm{DN}$ patients $(\mathrm{n}=26), 53.8 \%$ $(n=14)$ had a documented diagnosis of $\mathrm{p}-\mathrm{DN}$ by the referring physician, and $46.2 \%(\mathrm{n}=12)$ were identified by the pharmacists. A total of $95 \%$ ( 19 of 20$)$ of the patients treated for p-DN received adequate pharmacological agents, though suboptimal as per clinical guidelines. More than $50 \%$ of the patients used subtherapeutic doses of their medications. Gabapentin was the most frequently used medication in our population (65.4\%). Patients in rural South Carolina had a higher prevalence of DPN and p-DN with $>60 \%$ undocumented cases of $\mathrm{p}$-DN. More than $95 \%$ of treated patients did not receive optimum therapy according to AAN guidelines.

Keywords: polysensory neuropathy, pharmacist-led diabetes clinic, diabetes educator, gabapentin, chronic pain free clinic, pain, gabapentin

\section{Introduction}

Diabetes is emerging as an epidemic in western society. As of 2014, diabetes in the USA was estimated to be $9.3 \%$ of the population ( 29.1 million people), including 21 million diagnosed and 8.1 million undiagnosed cases. ${ }^{1}$ Type 2 diabetes represents $95 \%$ of all diabetes diagnoses. ${ }^{1,2}$ Diabetes has become even more pronounced in rural areas with a prevalence rate $17 \%$ higher than that in urban areas. ${ }^{3}$ Patients in rural areas are at a significant disadvantage because of decreased access to care, socioeconomic factors, and low health literacy. ${ }^{4,5}$ In fact, patients who reside in the southeastern region of the USA have a higher probability of being uninsured and living in rural areas, which also decreases their chances of accessing diabetic education. ${ }^{5,6}$ To add to this 
burden, patients in rural areas have higher poverty rates, decreased access to insurance, decreased household income, lower high school graduation rates, and higher mortality than those in urban area patients. ${ }^{5,6}$ All these disadvantages make diabetic patients in rural areas more vulnerable to develop diabetic comorbidities or complications. For example, there is a rising trend in diabetic patients in rural areas receiving primary care through the emergency department for diabetic complications such as foot ulcer and infections (a condition derived from diabetic peripheral neuropathy [DPN]), which increase health-care costs. ${ }^{6,7}$ The increase in hospital use could be attributed to increased distance to clinics, lack of adequate transportation, relative shortage of specialists and diabetes educators, cultural beliefs, and patient cost of care. ${ }^{5,6}$ It has been demonstrated that pharmacist-led free clinics in rural areas that provide educational programs to diabetic patients result in significant patient outcome improvements and financial savings. ${ }^{8}$ The current study focuses on painful diabetic neuropathy ( $\mathrm{pDN}$ ) in a rural diabetic population of South Carolina that is assisted by a pharmacist-led diabetes self-management education and support (DSEM/S) program.

Among US diabetic patients, an average of $28 \%-45 \%$ develops $\mathrm{DPN}^{9-11}$ and $11 \%-24 \%$ of them develop p-DN, of whom $39 \%$ of p-DN cases are left untreated. ${ }^{12-14}$ In addition, it has been reported that up to $61.5 \%$ of patients with type 2 diabetes are not documented as having DPN. ${ }^{15}$ However, the proportion of undocumented patients with p-DN is not known.

Patients who are left untreated for unregulated glycemic control have an increased likelihood of developing further damage to their peripheral sensory neurons, which could lead to ulcers and ultimately foot amputation. ${ }^{16} \mathrm{p}-\mathrm{DN}$ is a subtype of DPN. p-DN is characterized by prickling, stabbing, and burning sensations, which reflect the involvement of unmyelinated $\mathrm{C}$ fibers. ${ }^{6}, 16$ Treatment for $\mathrm{p}-\mathrm{DN}$ is complex due to the involvement of multiple pathways in its pathophysiology, and current therapy includes glucose control to prevent further progression and pain management. ${ }^{6}$ Treating p-DN has been challenging, with reduced patient satisfaction with pharmacological treatments and many inappropriate therapies. Furthermore, pain management with maximum pharmacological therapies produces mixed patient responses with goals of $30 \%-50 \%$ pain reduction in most patients. ${ }^{17-19}$ In fact, $>30 \%$ pain reduction is considered a successful treatment in patients with p-DN. ${ }^{18,19}$ Historically, it has been reported that a large proportion of patients with $\mathrm{p}-\mathrm{DN}$ do not receive pharmacological treatment or receive therapy with agents with no known efficacy in p-DN. ${ }^{18,20}$ Therefore, the access to treatment for $\mathrm{p}-\mathrm{DN}$ should be periodically assessed and documented, especially in rural areas where access to health care, in general, is diminished.

There is a gap in the current literature regarding the treatment of $\mathrm{p}$-DN in uninsured patients in underserved or rural areas. It is currently not well documented what pain treatment patients are receiving and how they are being treated compared to current guidelines. The current diabetic neuropathy treatment guidelines within the US are from the American Academy of Neurology (AAN) and the European Federation of the Neurological Societies (EFNS).

We hypothesize that uninsured patients from a rural area of the USA, more specifically, South Carolina, have a greater prevalence of $\mathrm{p}$-DN than currently available national average data and that a significant proportion of patients identified with p-DN by pharmacists in a Diabetes Self-management Education and Support in Type 2 Diabetes (DSME/S) program do not have a documented diagnosis upon clinician referral to the program. We also hypothesize that a significant proportion of these patients do not have optimal pharmacological treatments for p-DN. Following the documentation of a rural pharmacist-led DSME/S program that serves uninsured diabetic patients referred by primary physicians for educational purposes, our primary outcomes were the following: determine the prevalence of DPN and p-DN in this patient population, assess the proportion of DPN and p-DN documented and undocumented by physicians and pharmacists, and determine the proportion of patients with optimal and suboptimal pharmacological treatment for $\mathrm{p}-\mathrm{DN}$.

\section{Materials and methods Data source}

Presbyterian College School of Pharmacy (PCSP) Wellness Center is a pharmacist-run DSME/S program located in rural Laurens County, South Carolina. This program serves patients who are located in Laurens County or surrounding counties and referred by their primary physician. An initial assessment is performed on each patient that includes a comprehensive foot examination. We performed a retrospective chart review analysis to identify subjects (clinical records) with a diagnosis of type 2 diabetes using ICD-9 code 250.00. We used eligible clinical records registered in the PCSP Wellness Center electronic medical record (EMR) from September 1, 2014, to December 31, 2015. Data collected and documented included age, gender, race, weight, body mass index (BMI), presence of a foot examination, number of insensate points during foot examination, diagnosis of diabetic neuropathy, signs and symptoms of diabetic neuropathy, diagnosis of painful neuropathy, signs 
and symptoms of painful neuropathy, and medication. The Institutional Review Board (IRB) of Presbyterian College approved this study. All patients whose clinical records were accessed provided written informed consent. All efforts were made to protect patient identification and any sensitive and confidential patient information as required by Health Insurance Portability and Accountability Act of 1996 guidelines.

\section{Inclusion/exclusion criteria}

Clinical records of patients were evaluated using the following inclusion criteria: patient registered in PCSP Wellness Center database who received onsite diabetes education, diagnosis of type 2 diabetes, and $\geq 18$ years old. Exclusion criteria included patients not registered in PCSP Wellness Center EMR, patients not diagnosed with type 2 diabetes, patients enrolled in PCSP's telepharmacy-based diabetes education program, and no attendance of evaluation at Wellness Center. Patients with no current assessment from the Wellness Center were excluded since they would have no record of a foot examination or clinical evaluation from the pharmacists, which could skew data. Patients with no records of attendance were excluded from the study.

\section{Outcome measures}

The major outcomes were DPN and p-DN. Identification and diagnosis of DPN were primarily determined by the presence or absence of a previous diagnosis of DPN or $\mathrm{p}-\mathrm{DN}$ by the referring doctor, which was either specified on the patient chart or mentioned in doctor referral notes. In all cases, we determined whether the Wellness Center identified patients with DPN or p-DN, which was indicated by a diagnosis statement or comment of neuropathy or painful neuropathy on the letter to a physician, or in pharmacist recommendations. Pharmacists from the PCSP Wellness Center performed in all consenting patients a foot examination using Semmes-Weinstein monofilaments at 12 points on plantar (9) and dorsal (3) aspects of the foot as recommended by the American Diabetes Association for identification of diabetic neuropathy. ${ }^{21}$ The Semmes-Weinstein monofilament examination has been shown to serve as an independent predictor of risk of future foot ulcer and ultimately amputation. ${ }^{22}$ This examination is particularly useful for clinics in rural areas lacking adequate time, providers shortages, and monetary resources. The use of a $10 \mathrm{~g}$ (5.07) monofilament and the evaluation of 12 points on the plantar and dorsal aspects of both feet, including the great toes, third, and fifth metatarsal heads, has been demonstrated to provide $93 \%$ sensitivity and $100 \%$ specificity of the Semmes-Weinstein monofilament examination when compared to the gold standard, nerve conduction study. ${ }^{23-26}$ Therefore, these criteria were used in our assessment. The questionnaire was administered by a pharmacist who was a certified diabetes educator (CDE) or by a student pharmacist under direct pharmacist supervision during the initial wellness screening. The pharmacists were trained to perform monofilament foot examinations and assess patients for diabetic complications during training to become a CDE. The presence of at least one insensate point on the foot examination was used as indicative of the presence of peripheral neuropathy. This diagnostic threshold criterion has been demonstrated to be the most sensitive approach to determine DPN. ${ }^{23}$ Therefore, we determined whether the foot examination was positive (one or more insensate points) or negative (no insensate points). In addition to the foot examination, during the physical assessment of the patient, the pharmacist visually assessed each foot for abnormalities and made notes of ulcers, vascular warmth, or any other abnormalities. The pharmacists also administered a questionnaire to determine patient symptoms of DPN or p-DN. During the questionnaire, the pharmacist asked the patient for the presence of pain or paresthesia characteristic of any of the following keywords used to indicate DPN: tingling, prickling, needles, and numbness. In addition to above, the pharmacist also used keywords to indicate p-DN, which included pain, burning, and/or hurting in the lower or upper extremities, along with either an indication of DPN or a history of medication usage for DPN. ${ }^{6}$

Patients with other medical conditions that cause lower or upper extremity pain, such as arthritis, sciatica, edema, and fibromyalgia, and those who did not display any neurological manifestations consistent with DPN were not qualified as p-DN.

Using this information, we categorized these data into DPN or p-DN diagnosis by the referring physician (specific statement of diagnosis by the referring physician and medication treatment for $\mathrm{p}-\mathrm{DN}$ in the clinical record of the PCSP Wellness Center) and DPN or p-DN identification by the PCSP Wellness Center pharmacists (with no specific diagnosis determination by the referring physician).

\section{Demographics}

Age, weight, and BMI were taken as continuous variables. Gender was documented in the patient chart or self-reported by the patient. The race was optionally self-reported by the patient and classified as White, Black/African American, Hispanic, other (American Indian/Alaska Native, Mixed Race, Asian Indian, and Hawaiian/Pacific Islander), or unreported. 


\section{Medication assessments}

Medications were identified as a treatment in the clinical records. Patients with comorbidities that manifested signs and symptoms similar to DPN were excluded from the p-DN treatment analysis. Pain medications for comorbidities such as epilepsy, depression, arthritis, surgery, postherpetic neuralgia, and others that manifest similar to $\mathrm{p}$-DN were excluded. We used AAN's Treatment of Painful Diabetic Neuropathy Guideline as criteria for optimal, subtherapeutic, or improper medication management of $\mathrm{p}$-DN. Medications with AAN level A were considered optimal or therapeutic pharmacological management, medications with AAN level $B$ were considered suboptimal pharmacological management, and medications without AAN level A or B (level U) were considered improper treatment for $\mathrm{p}-\mathrm{DN}$. The medication dosages of patients were documented and compared to AANrecommended dosages for appropriateness.

\section{Statistical analysis}

After identifying DPN, patients were divided into two groups (DPN vs. no DPN). Proportion of patients with DPN in the study population and proportion of patients classified as $\mathrm{p}-\mathrm{DN}$ and without $\mathrm{p}-\mathrm{DN}$ among those with DPN were calculated. Independent $t$-Tests for continuous variables (age, weight, and BMI) and chi-square or Fisher's exact tests for categorical variables (race and gender) compared the characteristics of study population (DPN vs. no DPN) and characteristics of the subpopulation with DPN (p-DN vs. without $\mathrm{p}-\mathrm{DN}$ ). Pain medication use patterns were described by proportions. Mann-Whitney $U$-test compared the pain medication doses (optimal use vs. suboptimal use) in patients with $\mathrm{p}-\mathrm{DN}$. The statistical significance was defined at $p<0.05$. All analyses were performed using the software Statistical Analysis Software and Prism GraphPad.

\section{Results}

Of 132 patient records, 111 patients met inclusion criteria and were used for further analysis (Figure 1). Table 1 shows there were no significant differences in race, gender, age, weight, or BMI between patient with DPN and those without DPN. Table 2 displays the demographic characteristics of patients with DPN (p-DN vs. without p-DN). The p-DN group showed higher proportion from the male group (62.2\% vs. $18.6 \%$, $p<0.001)$ and less weight $(90.6 \mathrm{~kg}$ vs. $102.6 \mathrm{~kg}, p=0.047)$ than the without $\mathrm{p}-\mathrm{DN}$ group.

Among 111 eligible patients, we identified 69 patients $(62.2 \%)$ with DPN. From the total DPN subpopulation $(n=69), 29 \%(n=20)$ had a documented diagnosis of DPN by the referring physician, and $71 \%(n=49)$ did not have any indication in the clinical records (and referral notes) of DPN

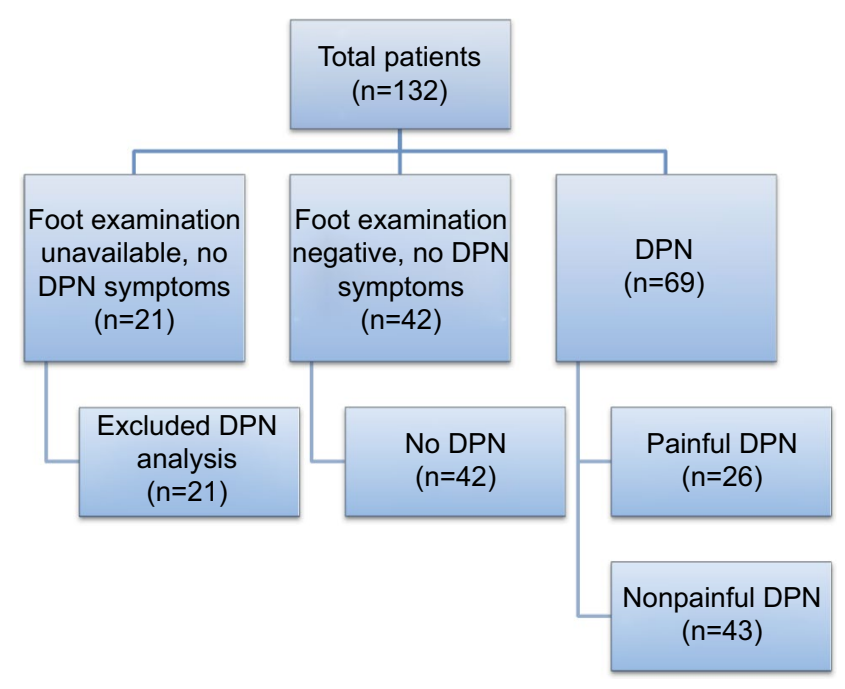

Figure I Identification of DPN and p-DN in PCSP Wellness Center diabetic patients.

Abbreviations: DPN, diabetic peripheral neuropathy; p-DN, painful diabetic neuropathy; PCSP, Presbyterian College School of Pharmacy.

Table I Demographic characteristics of the studied population $(n=I I I)$

\begin{tabular}{|c|c|c|c|c|}
\hline Characteristics & All $(n=I I I), n(\%)$ & DPN (n=69), n (\%) & No DPN (n=42), n (\%) & $p$ \\
\hline White & $53(47.7)$ & $31(44.9)$ & $22(52.4)$ & 0.703 \\
\hline Black/AA & $37(33.3)$ & $24(34.8)$ & $13(31.0)$ & \\
\hline Hispanic & $4(3.6)$ & $3(4.3)$ & I (2.4) & \\
\hline Asian Indian & $1.8(2)$ & $\mathrm{I}(\mathrm{I} .5)$ & I (2.4) & \\
\hline Hawaiian/Pacific Islander & I (0.9) & 0 & $\mathrm{I}(2.4)$ & \\
\hline Unreported & $14(12.6)$ & $10(14.5)$ & $4(9.5)$ & \\
\hline \multicolumn{5}{|l|}{ Gender } \\
\hline Male & $46(4 I .4)$ & $26(37.7$ & $20(47.6)$ & 0.302 \\
\hline \multirow[t]{2}{*}{ Female } & $65(65)$ & $43(62.3)$ & $22(52.4)$ & \\
\hline & Mean (SD) & Mean (SD) & Mean (SD) & \\
\hline Age (years) & $55.8(11.0)$ & $56.23(10.3)$ & $55.14(12.0)$ & 0.245 \\
\hline Weight (kg) & $98.9(26.1)$ & $98.1(24.2)$ & $100.2(28.9)$ & 0.954 \\
\hline BMI $\left(\mathrm{kg} / \mathrm{m}^{2}\right)$ & $34.8(7.7)$ & $34.7(7.5)$ & $35.1(7.9)$ & 0.799 \\
\hline
\end{tabular}

Abbreviations: AA, African American; BMI, body mass index; DPN, diabetic peripheral neuropathy; SD, standard deviation. 
Table 2 Demographic characteristics of the $p-D N(n=26)$ population vs. DPN without $\mathrm{p}-\mathrm{DN}$

\begin{tabular}{|c|c|c|c|}
\hline Characteristics & $\begin{array}{l}\text { p-DN }(n=26), \\
n(\%)\end{array}$ & $\begin{array}{l}\text { DPN without p-DN } \\
(n=43), n(\%)\end{array}$ & $p$ \\
\hline White & II (42.3) & $20(45.5)$ & 0.630 \\
\hline Black/AA & $8(30.7)$ & $16(37.2)$ & \\
\hline Hispanic & I (3.8) & $2(4.7)$ & \\
\hline Asian Indian & I (3.8) & 0 & \\
\hline Race unreported & $5(19.2)$ & $5(11.6)$ & \\
\hline \multicolumn{4}{|l|}{ Gender } \\
\hline Male & $18(69.2)$ & $8(18.6)$ & $<0.001$ \\
\hline \multirow[t]{2}{*}{ Female } & $8(30.8)$ & $35(8 I .4)$ & \\
\hline & Mean (SD) & Mean (SD) & \\
\hline BMI $\left(\mathrm{kg} / \mathrm{m}^{2}\right)$ & $33.4(6.0)$ & $35.4(8.3)$ & 0.301 \\
\hline Age (years) & $54.3(8.4)$ & $57.4(11.3)$ & 0.226 \\
\hline Weight (kg) & $90.6(21.1)$ & $102.6(25.0)$ & 0.047 \\
\hline
\end{tabular}

Abbreviations: AA, African American; BMI, body mass index; DPN, diabetic peripheral neuropathy; $\mathrm{p}-\mathrm{DN}$, painful diabetic neuropathy; SD, standard deviation.

by the primary physicians but were identified by the PCSP Wellness Center pharmacists (Table 3).

In our patient population ( $\mathrm{n}=111$ with inclusion criteria), we identified 26 patients $(23.4 \%)$ with p-DN, which represents $37.7 \%$ of the DPN population $(n=69)$. In our $\mathrm{p}-\mathrm{DN}$ patient population $(n=26), 46.2 \%(n=12)$ had a documented diagnosis of $\mathrm{p}-\mathrm{DN}$ by the referring physician, and $53.8 \%$ $(n=14)$ did not have any indication of $\mathrm{p}-\mathrm{DN}$ in the clinical records (and referral notes) of p-DN by the primary physicians but were screened positively for p-DN by the PCSP Wellness Center pharmacists (Table 3).

In our $\mathrm{p}$-DN population $(\mathrm{n}=26), 76.9 \%(\mathrm{n}=20)$ were prescribed metformin and $100 \%(n=26)$ were prescribed a medication with an indication for the treatment of diabetes. All the patients diagnosed with $\mathrm{p}-\mathrm{DN}$ by the primary physician $(n=12)$ were prescribed treatment for pain. From the patients with no record indication of $\mathrm{p}-\mathrm{DN}$ by the primary physician (identified by the pharmacists, $53.8 \%, \mathrm{n}=14$ ), only eight patients $(57.1 \%)$ were prescribed treatment for pain, leaving the remaining six patients $(42.9 \%)$ with no documented pain treatment. In total, patients presenting with $\mathrm{p}-\mathrm{DN}, 76.9 \%(\mathrm{n}=20)$ received medication therapy for $\mathrm{p}-\mathrm{DN}$ symptoms, and $23.1 \%(\mathrm{n}=6)$ did not receive therapy at all for p-DN (Table 4).

Table 3 Proportion of diagnosis or identification of DPN and $\mathrm{p}$-DN by health professional

\begin{tabular}{lll}
\hline Health professional & DPN, n (\%) & p-DN, n (\%) \\
\hline By PharmD & $49(71)$ & $14(53.8)$ \\
By MD & $20(29)$ & $12(46.2)$ \\
Total & $69(100)$ & $26(100)$ \\
\hline
\end{tabular}

Abbreviations: DPN, diabetic peripheral neuropathy; p-DN, painful diabetic neuropathy; PharmD, Doctor of Pharmacy; MD, Doctor of Medicine.
Table 4 Proportion of pain medication use in patients with p-DN $(n=26)$

\begin{tabular}{llll}
\hline p-DN population & N (\%) & $\begin{array}{l}\text { Dx by MD, } \\
\text { n (\%) }\end{array}$ & $\begin{array}{l}\text { ID by PharmD, } \\
\text { n (\%) }\end{array}$ \\
\hline With pain medication & $20(76.9)$ & $12(46.1)$ & $8(30.8)$ \\
Without pain medication & $6(23.1)$ & 0 & $6(23.1)$ \\
Total & $26(100)$ & $12(46.1)$ & $14(53.9)$ \\
\hline
\end{tabular}

Abbreviations: $\mathrm{Dx}$, diagnosis; ID, identified; $\mathrm{p}-\mathrm{DN}$, painful diabetic neuropathy; PharmD, Doctor of Pharmacy; MD, Doctor of Medicine.

When specific medication therapies were reviewed in our p-DN population ( $\mathrm{n}=26$, Table 5), gabapentin (suboptimal therapy by AAN guidelines) was the most frequently used medication in our studied $\mathrm{p}$-DN population (17 patients, $65.4 \%$, Table 5). Figure 2 describes the patterns of combination of pain medication use in patients with $\mathrm{p}$-DN. The major findings described in Figure 2 are the following: 1) gabapentin plus opioids were the most frequent combination therapy $(n=8), 2)$ gabapentin plus antidepressants were the second most frequently used combination within $\mathrm{p}-\mathrm{DN}$ patients $(\mathrm{n}=5)$,

Table 5 Types of pain medication use in patients with $\mathrm{p}-\mathrm{DN}$ $(\mathrm{n}=26)$

\begin{tabular}{ll}
\hline Medication $^{\mathbf{a}}$ & Number of patients (\%) \\
\hline Pregabalin & $\mathrm{I}(3.8)$ \\
Gabapentin & $17(65.4)$ \\
Antidepressants & $8(30.7)$ \\
Opioids & $7(34.6)$ \\
Other agents & $4(15.4)$ \\
\hline
\end{tabular}

Note: asome patients received more than one drug.

Abbreviation: pDN, painful diabetic neuropathy.

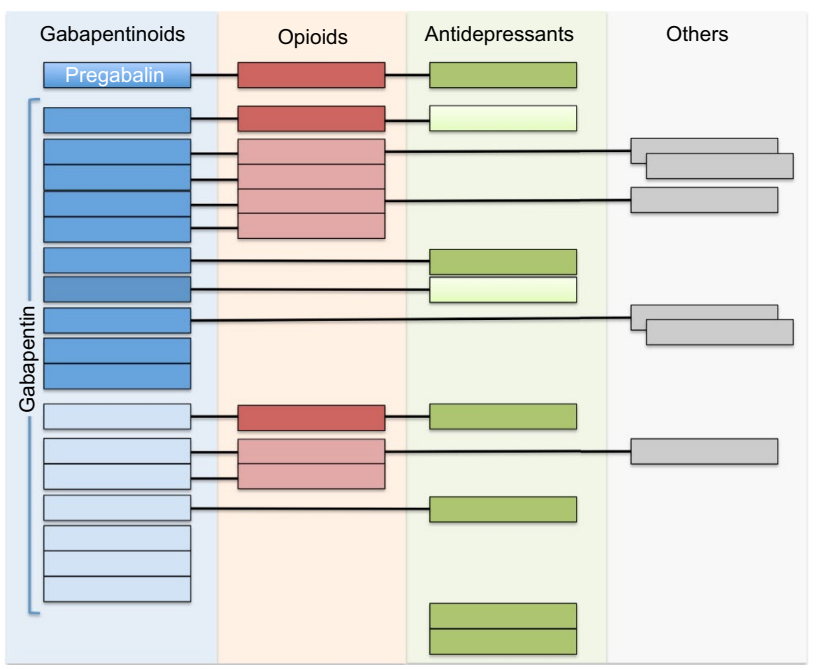

Figure 2 Diagram of combination of medications used by patient.

Notes: Dark blue (gabapentinoids), dark green (antidepressants), and dark red (opioids) implies AAN-recommended doses. Light blue, light green, and light red implies lower doses recommended by AAN. Gray represents other medications (i.e., tizanidine, meloxicam, and cyclobenzaprine). Linked lines indicate concomitant use. Abbreviation: AAN, American Academy of Neurology. 
3) gabapentin was given at subtherapeutic dosages in $41.2 \%$ of cases $(n=10 / 17), 4)$ opioids were given at subtherapeutic dosages in $66.7 \%$ of cases $(n=6 / 9), 5)$ antidepressants were given at subtherapeutic dosages in $12.5 \%$ of cases $(n=2 / 8)$, and 6) when taking into consideration all the treatment combinations, $65 \%(n=13 / 29)$ of patients with treatment for $\mathrm{p}-\mathrm{DN}$ received subtherapeutic dosages in at least one drug.

Among patients with gabapentin plus opioids, four used tramadol (prn), two hydrocodone/acetaminophen, and two oxycodone/acetaminophen. From the patients with gabapentin plus antidepressants, three used duloxetine (one with oxycodone/acetaminophen), one used amitriptyline (plus oxycodone/acetaminophen), and one used nortriptyline. From the patients with gabapentin and other medications, three used cyclobenzaprine (one with hydrocodone/acetaminophen and one with meloxicam). Pregabalin (optimal therapy by AAN guidelines) was used by one patient (3.8\%, Table 5). This patient also used hydrocodone/acetaminophen and amitriptyline (Figure 2). In summary, gabapentinoids (gabapentin + pregabalin) were used in $69.2 \%$ of cases $(n=18)$, opioids in $34.7 \%(\mathrm{n}=9)$, antidepressants in $30.7 \%(\mathrm{n}=8)$, and other agents (cyclobenzaprine, tizanidine, or meloxicam) in 15.3\% $(n=4)$ of cases.

In total (Figure 2), opioids were used in combination with gabapentinoids by nine patients ( $34.6 \%$ ) of the $\mathrm{p}$-DN population when including hydrocodone $(n=3$, drug not included in the recommended drugs in the AAN guidelines). We found that amitriptyline was used by four patients (15.4\%), two as monotherapy (suboptimal therapy), and nortriptyline was used by one patient (3.8\%) of the p-DN population. Duloxetine was used by three patients (11.5\%) of the p-DN population. When these findings were compared to the AAN Guidelines for the treatment of painful DPN, ${ }^{13}$ we uncovered that $95 \%$ (19 of 20) of the patients treated for p-DN received suboptimal treatment (gabapentin or antidepressants, alone or in combination with other agent) and not optimal therapy (first-line treatment, namely, pregabalin).

Analysis of individual pharmacological agents revealed that of 17 patients using gabapentin, 10 patients $(58.8 \%)$ received the medication in the optimum dose range (900$3,600 \mathrm{mg} / \mathrm{d})$ and $7(41.2 \%)$ received subtherapeutic doses (<900 mg/d, Figure 3). The average (mean) dose of gabapentin in the optimum dose range was significantly different than the dose in the subtherapeutic dose range $(p<0.05$, Figure 4$)$, $1,350$ (95\% confidence interval: $981.7-1,718)$ vs. $360(95 \%$ confidence interval: 193.4-526.6).

Among the patients receiving optimal doses of gabapentin, two patients used gabapentin alone, and eight patients

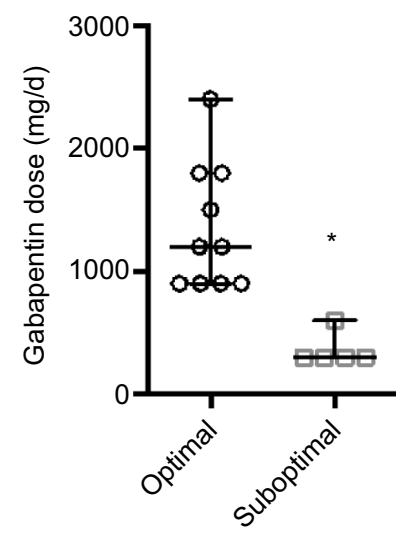

Types of therapeutic doses

Figure 3 Comparison of doses between patients receiving AAN-recommended doses of gabapentin (optimal) and doses below AAN recommendation (suboptimal). Notes: ${ }^{*} p<0.05$ between groups, Mann-Whitney $U$-test. Data are represented as median with range.

Abbreviation: AAN, American Academy of Neurology.

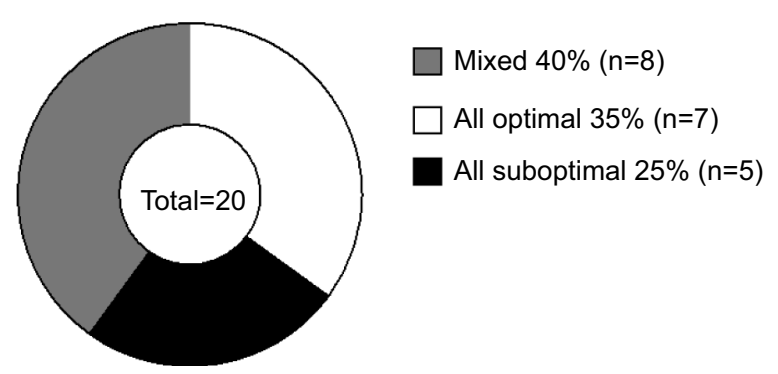

Figure 4 Proportion of p-DN patients receiving drug combinations with optimal, suboptimal, or partially optimal (mixed) doses.

Abbreviation: $\mathrm{p}-\mathrm{DN}$, painful diabetic neuropathy.

used other drugs (five used opioids, three antidepressants, two meloxicam, and three a muscle relaxant). From patients with optimal doses of gabapentin plus other pain medications, four of them used three drugs in total, and four used two drugs in total. Among the patients receiving suboptimal doses of gabapentin, three patients used gabapentin alone, and four patients used other drugs (three used opioids, two antidepressants, and one a muscle relaxant). From patients with suboptimal doses of gabapentin plus other pain medications, two of them used three drugs in total, and two used two drugs in total (Figure 2).

Our study reveals that only seven patients with treatment for $\mathrm{p}-\mathrm{DN}$ ( $\mathrm{n}=20$ total) received treatment with recommended doses for all drugs used as follows: one patient with pregabalin plus an opioid and an antidepressant, one patient with gabapentin plus an antidepressant, two patients with only one antidepressant, and three patients with only gabapentin. Our study also uncovers that at least five patients 
Table 6 Number of pain medication use in patients with p-DN $(\mathrm{n}=26)$

\begin{tabular}{ll}
\hline Number of medications & Number of patients (\%) \\
\hline 0 & $6(23.1)$ \\
1 & $7(26.9)$ \\
2 & $6(23.1)$ \\
3 & $6(23.1)$ \\
4 & $1(3.8)$ \\
\hline Abbreviation: pDN, painful diabetic neuropathy.
\end{tabular}

with treatment for $\mathrm{p}-\mathrm{DN}$ ( $\mathrm{n}=20$ total) are receiving drugs at suboptimal doses as follows: two patients with gabapentin plus an opioid, and three patients with only gabapentin (Figure 4). Additionally, eight patients with treatment for $\mathrm{p}-\mathrm{DN}$ $(n=20$ total) received at least one drug at suboptimal doses in combination with one or two drugs at recommended doses as follows: one patient with optimal doses of gabapentin and an opioid and suboptimal doses of an antidepressant, four patients with optimal doses of gabapentin and suboptimal doses of an opioid, one patient with optimal doses of an antidepressant and suboptimal doses of an opioid plus gabapentin, one patients with optimal doses of an antidepressant plus suboptimal doses of gabapentin, and one patients with optimal doses of gabapentin plus suboptimal doses of an antidepressant (Figure 4).

Table 6 displays the number of patients receiving zero, one, two, three, or four medications that were taken to treat p-DN. Among 26 patients with p-DN, 23.1\% did not receive a pain medication and $73.1 \%$ received at least one but less than four pain medications.

\section{Discussion}

Our study shows that the patients from the PCSP Wellness Center DSME/S program had a prevalence of DPN that is higher than previously published average in the US (67\% vs. $28 \%-45 \%$, respectively). ${ }^{9-11}$ The patient population within our study had a p-DN prevalence of $23.4 \%$, which is near the upper range of $11 \%-24 \%$, previously reported from multiple trials in the USA. ${ }^{12-14,27}$ This trend could be explained by the rural nature of Laurens County, which implies decreased access to health care, decreased health literacy, and lack of health insurance. ${ }^{5}$ Patients who are referred to diabetic education courses, such as those at PCSP Wellness Center, are usually those with poor glycemic control needing personal assistance and identified by primary care physicians. This provides an additional explanation to our rather high prevalence of DPN and p-DN in this population.

A significantly greater percentage of men in our study suffer $\mathrm{p}-\mathrm{DN}$ when compared to women. These data contradict previous literature in which more women reported symptoms of p-DN. ${ }^{28}$ Whether these differences are due to the geographical locations of the studies (UK vs. USA) or the rural nature of this study population is something that we cannot determine with our available data. In addition, there was a statistical significant difference in weight between patients with p-DN vs. nonpainful DPN. Patients in the p-DN group had a mean weight of $90.7 \mathrm{~kg}$ compared to $102.6 \mathrm{~kg}$ for patient with nonpainful DPN. This could be due to the fact that patients with nonpainful DPN may be affected with a more advanced deficit in tactile sensitivity (neuropathy with no pain) that is associated with a higher weight, in comparison with patients with lower weight and p-DN that may be associated with a less severe stage of DPN (less compromised sensory fibers).

Our study confirmed previous literature with $>61.5 \%$ (72.5\%) of our patient population (type 2 diabetes) not having documentation of DPN in referring patient information from their primary physician. ${ }^{15}$ Additionally, our study uncovers that $53.8 \%$ of our patient population did not have documentation of $\mathrm{p}-\mathrm{DN}$. In our study, some patients $(\mathrm{n}=8)$ received treatment that could be indicated for $\mathrm{p}-\mathrm{DN}$ without having a diagnosis of $\mathrm{p}-\mathrm{DN}$ from the primary physician. This can be potentially explained by a lack of communication and incomplete medication information being exchanged between the referring physician and PCSP Wellness Center. Therefore, the most likely rate of physician diagnosed p-DN cases in our population is $76.9 \%(n=20)$, leaving $23.1 \%(n=6)$ identified by the pharmacists. A possible alternative explanation is that medications used off-label for $\mathrm{p}-\mathrm{DN}$ could have been for a different undiagnosed or undocumented condition (depression, fibromyalgia, and other conditions with FDA indications). In any case, our study uncovers some documentation deficiencies that may exist between primary care physician referral notes and additional health-care providers. The identification of these gaps in documentation is crucial for the proper treatment, follow-up of disease progression, and/ or assessment of drug adherence. Our study demonstrates that there is still room for improvement for communication throughout all health-care settings to prevent patients from going undiagnosed and untreated for debilitating conditions. There is a need for a communication protocol that informs care providers of the current and correct diagnosis of patients served at different locations, such as EMRs and ICD-10 codes. Our study shows how there are multiple steps in identifying, diagnosing, treating, and educating patients with DNP and p-DN.

Pharmacist-led free clinics has been shown to provide significant reductions from baseline in $\mathrm{HbA} 1 \mathrm{c}$ values, systolic 
blood pressure, and triglyceride levels, which equates to health-care savings of $\$ 1,118$ per patient who had a decrease of $>1 \%$ in $\mathrm{HbA}(1 \mathrm{c})$ value. ${ }^{8}$ Our study is in line with this notion. The diabetic education program in the pharmacist-led Wellness Center at PCSP was robust enough to identify potential patients with DPN and p-DN who were not diagnosed by primary care physicians. Of note, our study uncovers that $>20 \%$ of p-DN patient (6 out of 26 patients) were not documented as receiving pharmacological therapy to manage their painful symptoms, and this finding coincided with the lack of documentation of $\mathrm{p}-\mathrm{DN}$ previous to the PCSP Wellness Center evaluation in all these cases. Each patient's primary care provider was emailed the results of the patient evaluations and findings while enrolled in PCSP Wellness Center. Our study shows that pharmacist-led diabetic education programs can collaborate with physicians to increase awareness of patient with p-DN within rural populations. We recognize that the size of our studied p-DN population is modest (26); however, we were able to uncover meaningful information, like the abovementioned proportion of patients without diagnose and treatment for this condition.

This study was able to shed light on the use of various medication regimens for uninsured patients within a rural community. According to the AAN, the first-line medication (level A) for the treatment of $\mathrm{p}-\mathrm{DN}$ is pregabalin, an anticonvulsant. ${ }^{19}$ We found that over $96 \%(n=19)$ of patients with $\mathrm{p}-\mathrm{DN}$ were not being treated in accordance with AAN level A treatment guidelines (pregabalin). ${ }^{19}$ The decreased income of patients in rural areas offers the simplest explanation for this circumstance. As of August 2016, the cost for pregabalin listed at wholesale acquisition cost ranges from $\$ 517.54$ to $\$ 724.56$ per package size. ${ }^{29}$ According to the AAN guidelines, pregabalin is supported by four class 1 trials that show superior reductions in pain ranging 10\%-50\% compared to placebo. ${ }^{19}$ In addition, pregabalin produces an improvement in the quality of life such as social functioning, mental health, bodily pain, and vitality with reductions in sleep interference. ${ }^{19,30}$ With patients unable to afford AAN level A, alternatives such as gabapentin (primarily), amitriptyline, and/or opioids become attractive options to treat patients with low income with p-DN in rural areas. Gabapentin was used in $>60 \%$ of our p-DN patients. Gabapentin was in fact the most used medication in an outpatient population. The AAN states that gabapentin can achieve pain reductions of $11 \%$ and improvement in mental health and vitality. ${ }^{19}$ Additional literature on gabapentin shows that gabapentin produces $50 \%$ pain intensity reduction in $35 \%$ of patients compared to $20 \%$ in placebo group in chronic neuropathic pain and fibromyalgia. ${ }^{31}$ The cost of gabapentin makes it attractive for $\mathrm{p}$-DN treatment with wholesale acquisition cost package prices as low as $\$ 2.28$ as of August $2016 .{ }^{29}$ Our data suggest that the primary care prescribers in this rural area in South Carolina could have taken these factors into consideration and, therefore, chose gabapentin for this particular population. Even though gabapentin as the principal medication for the treatment of $\mathrm{p}$-DN seems an adequate option for p-DN patients with limited financial resources, our data demonstrate that 7 out of 17 patients with gabapentin were receiving suboptimal doses $(<900 \mathrm{mg} / \mathrm{d})$.

Opioids have AAN level B evidence; however, there is very limited information that supports the use of morphine, tramadol, or oxycodone for p-DN. ${ }^{19}$ Opioids have been shown to have a more therapeutic value as add-on therapy for those who fail previous anticonvulsants and antidepressant medications. ${ }^{19,32,33}$ The recommended use of opioids as a level B by the AAN is also in line with the multiple side effects associated with these drugs, namely low tolerability, abuse potential, constipation, sedation, vomiting, etc. ${ }^{17-19}$ Similarly, the EFNS guidelines recommend opioids as a second line and gabapentin as the first line. ${ }^{34}$ Similarly, the Canadian Pain Society recommend opioids as third-line agents and gabapentin as the first line for the treatment of neuropathic pain. ${ }^{35}$ The American Pain Society states that the chronic opioid therapy could be an option after other therapies indicated for neuropathic pain have been tried, but only for carefully monitored patients. ${ }^{36}$ Our findings are in line with these guidelines since opioids were used in $>34 \%$ of the study population, in which they were most frequently used in combination with gabapentin plus antidepressant $62.5 \%(n=5)$ of their total use (Figure 2$)$. In our studied population, four out of nine $\mathrm{p}-\mathrm{DN}$ patients with opioids were prescribed prn, indicating its adjuvant use. However, all these four patients were prescribed suboptimal doses of opioids, based on the ANN guidelines. From these four patients, two were receiving optimal doses of gabapentin.

Antidepressants have AAN level B evidence. ${ }^{19}$ Antidepressant medications were used by $30.7 \%(n=8)$ and all of them were receiving AAN-recommended doses. Antidepressants were most frequently used in combination with gabapentin $(n=5)$. Two of these five patients were receiving suboptimal doses of gabapentin. Two patients were receiving antidepressants as the only pharmacological treatment. Antidepressants provide an additional cost-efficient treatment option for uninsured p-DN patients. Both amitriptyline and nortriptyline are generic options, while duloxetine has, as of March 2016, became available as generic. Interestingly, in Europe, EFNS 
guidelines give antidepressants level A, with evidence of $50 \%$ pain reduction. ${ }^{34}$ Nevertheless, previous literature from a Denmark study supports that tricyclic antidepressants relieve one in every two to three patients with p-DN and work better than options such as tramadol and oxycodone. ${ }^{37}$

Our data indicate that only 7 patients out of 20 p-DN patients with treatment have optimal doses of all the prescribed drugs. This means that at least 13 patients were under partially optimal or suboptimal doses of their prescribed medication for $\mathrm{p}-\mathrm{DN}$. Since we did not have access to pain intensity values (the free clinic records did not included this information), we cannot accurately assess the efficacy of these drug regimens to manage patients' pain in our population. It is likely that an ineffective pain control was the reason of having multiple drugs. The fact that some of these drugs were at suboptimal doses suggests that these patients may not be receiving adequate pain management, despite their multiple drug regimen. Therefore, our data suggest that 13 out of 20 patients with p-DN medication are under a suboptimal pharmacological pain treatment. The records evaluated in this study did not show any indication of a treatment regime aimed to find an optimal dose using small dose escalations, which rules out the possibility that some patients with low doses (i.e., for gabapentin) were under a titration process. It is worth noting that the AAN guidelines for the treatment of $\mathrm{p}-\mathrm{DN}$ determine $900 \mathrm{mg} / \mathrm{d}$ (tid) as the effective threshold dose for gabapentin (threshold that used in this study), in comparison with other guidelines or studies (IASP guidelines and Rosenberg and Watson use $1,800 \mathrm{mg} / \mathrm{d}$ ). ${ }^{38,39}$ Therefore, the literature indicates that for gabapentin, a range of $900-3,600 \mathrm{mg} / \mathrm{d}$ should be used to treat mild to severe $\mathrm{p}-\mathrm{DN}$.

We recognize that one of the limitations of this study include small sample size. This limitation does not allow us to associate risk factors with the lack of identification or diagnosis of DPN or p-DN and with the prescription of optimal or suboptimal pharmacological treatments. Our future studies will include a sample size that allows us to perform this analysis. However, despite this small number of cases, our study uncovered relevant gaps and deficiencies in the documentation or identification of DPN and p-DN and treatments of patients with $\mathrm{p}-\mathrm{DN}$ in a small rural area of the USA. These findings could guide other health providers in small rural areas to conduct similar research or monitor more closely their protocols. In addition, another limitation of our study is a potential lack of complete documentation from referring physicians that could mask the number of patients with a diagnosis upon referral to the free clinic. Additionally, our study relied on referring documentation and chart data obtained by pharmacists that included subjective information (i.e., pain, tingling, etc.) that may not translate to different patient populations or study designs. Even though the protocol used by the pharmacist was sufficiently sensitive to identify signs and symptoms of diabetic neuropathy and painful neuropathy, the lack of an international-validated questionnaire related to the intensity of pain or the efficacy of pain management is another limitation. This is valuable information that could be easily obtained from patients with p-DN. Our study identified this as an area of improvement and has since incorporated a validated questionnaire into the procedures of the PCSP Wellness Center.

Future directions of this study are to assess the outcomes of individual pain therapies in patients in rural areas using an international-validated diabetic neuropathy questionnaire. In addition, we plan to collect prevalence of DPN and p-DN in other rural areas across South Carolina and potentially the USA or other countries.

\section{Conclusion}

Our study shows that patients enrolled in PCSP Wellness Center's diabetes education program rural Laurens, South Carolina, had a high prevalence of $23.4 \%$ of p-DN (compared to national average of $17.5 \%[11 \%-24 \%])$. Our study also shows that documentation of $\mathrm{p}-\mathrm{DN}$ by primary care providers appears to be lacking or inconsistent, and a convenient and reliable method is warranted in rural settings. Additionally, this patient population appears to be receiving mostly suboptimal pharmacological therapy based on the AAN guidelines for the treatment of $\mathrm{p}-\mathrm{DN}$. Pharmacist-led DSME/S programs are in a position to reduce the percentage of patients being undiagnosed and untreated or suboptimally treated for $\mathrm{p}-\mathrm{DN}$ in rural communities affected by lack of access to health care.

\section{Acknowledgments}

The authors would like to acknowledge the funding provided by the Rita Allen Foundation and American Pain Society Award (EAR-S), Small Pharmacy Awards for Research and Collaboration (EAR-S), and Presbyterian College School of Pharmacy Pharmacy Research Summer Internship 2015 (CM-V and JP).

\section{Disclosure}

The authors report no conflicts of interest in this work.

\section{References}

1. Prevention CfDCa. National Diabetes Statistics Report: Estimates of Diabetes and Its Burden in the United States. Atlanta, GA: U.S. Department of Health and Human Services; 2014. 
2. Defronzo RA. Banting Lecture. From the triumvirate to the ominous octet: a new paradigm for the treatment of type 2 diabetes mellitus. Diabetes. 2009;58(4):773-795.

3. Massey CN, Appel SJ, Buchanan KL, Cherrington AL. Improving diabetes care in rural communities: an overview of current initiatives and a call for renewed efforts. Clin Diab. 2010;28(1):20-27.

4. Brown-Guion SY, Youngerman SM, Hernandez-Tejada MA, Dismuke CE, Egede LE. Racial/ethnic, regional, and rural/urban differences in receipt of diabetes education. Diabetes Educ. 2013;39(3):327-334.

5. Tonks SA, Makwana S, Salanitro AH, et al. Quality of diabetes mellitus care by rural primary care physicians. J Rural Health. 2012;28(4):364-371.

6. Tesfaye S, Selvarajah D. Advances in the epidemiology, pathogenesis and management of diabetic peripheral neuropathy. Diabetes Metab Res Rev. 2012;28(suppl 1):8-14.

7. Zhao Y, Ye W, Le TK, Boye KS, Holcombe JH, Swindle R. Comparing clinical and economic characteristics between commercially-insured patients with diabetic neuropathy and demographically-matched diabetic controls. Curr Med Res Opin. 2009;25(3):585-597.

8. Sease JM, Franklin MA, Gerrald KR. Pharmacist management of patients with diabetes mellitus enrolled in a rural free clinic. Am J Health Syst Pharm. 2013;70(1):43-47.

9. Dyck PJ, Kratz KM, Lehman KA, et al. The Rochester Diabetic Neuropathy Study: design, criteria for types of neuropathy, selection bias, and reproducibility of neuropathic tests. Neurology. 1991;41(6):799-807.

10. Harris M, Eastman R, Cowie C. Symptoms of sensory neuropathy in adults with NIDDM in the U.S. population. Diabetes Care. 1993;16(11):1446-1452.

11. Gregg EW, Sorlie P, Paulose-Ram R, et al. Prevalence of lower-extremity disease in the US adult population $\geq 40$ years of age with and without diabetes: 1999-2000 national health and nutrition examination survey. Diabetes Care. 2004;27(7):1591-1597.

12. Hartsfield CL, Korner EJ, Ellis JL, Raebel MA, Merenich J, Brandenburg N. Painful diabetic peripheral neuropathy in a managed care setting: patient identification, prevalence estimates, and pharmacy utilization patterns. Popul Health Manag. 2008;11(6):317-328.

13. Schmader KE. Epidemiology and impact on quality of life of postherpetic neuralgia and painful diabetic neuropathy. Clin J Pain. 2002;18(6):350-354.

14. Daousi C, MacFarlane IA, Woodward A, Nurmikko TJ, Bundred PE, Benbow SJ. Chronic painful peripheral neuropathy in an urban community: a controlled comparison of people with and without diabetes. Diabet Med. 2004;21(9):976-982.

15. Ziegler D, Strom A, Lobmann R, Reiners K, Rett K, Schnell O. High prevalence of diagnosed and undiagnosed polyneuropathy in subjects with and without diabetes participating in a nationwide educational initiative (PROTECT study). J Diabetes Complications. 2015;29(8):998-1002.

16. Albers JW, Pop-Busui R. Diabetic neuropathy: mechanisms, emerging treatments, and subtypes. Curr Neurol Neurosci Rep. 2014;14(8):473.

17. Callaghan BC, Cheng HT, Stables CL, Smith AL, Feldman EL. Diabetic neuropathy: clinical manifestations and current treatments. Lancet Neurol. 2012;11(6):521-534.

18. Lindsay TJ, Rodgers BC, Savath V, Hettinger K. Treating diabetic peripheral neuropathic pain. Am Fam Physician. 2010;82(2):151-158.

19. Bril V, England J, Franklin GM, et al. Evidence-based guideline: treatment of painful diabetic neuropathy: report of the American Academy of Neurology, the American Association of Neuromuscular and Electrodiagnostic Medicine, and the American Academy of Physical Medicine and Rehabilitation. Neurology. 2011;76(20):1758-1765.

20. Wong MC, Chung JW, Wong TK. Effects of treatments for symptoms of painful diabetic neuropathy: systematic review. BMJ. 2007; 335(7610):87.
21. Boulton AJ, Vinik AI, Arezzo JC, et al. Diabetic neuropathies: a statement by the American Diabetes Association. Diabetes Care. 2005;28(4):956-962.

22. Feng Y, Schlosser FJ, Sumpio BE. The Semmes Weinstein monofilament examination is a significant predictor of the risk of foot ulceration and amputation in patients with diabetes mellitus. JVasc Surg. 2011;53(1): 220-226.e1-5.

23. Feng Y, Schlosser FJ, Sumpio BE. The Semmes Weinstein monofilament examination as a screening tool for diabetic peripheral neuropathy. J Vasc Surg. 2009;50(3): 675-682,682.e1.

24. Lee S, Kim H, Choi S, Park Y, Kim Y, Cho B. Clinical usefulness of the two-site Semmes-Weinstein monofilament test for detecting diabetic peripheral neuropathy. J Korean Med Sci. 2003;18(1):103-107.

25. McGill M, Molyneaux L, Spencer R, Heng LF, Yue DK. Possible sources of discrepancies in the use of the Semmes-Weinstein monofilament. Impact on prevalence of insensate foot and workload requirements. Diabetes Care. 1999;22(4):598-602.

26. Smieja M, Hunt DL, Edelman D, Etchells E, Cornuz J, Simel DL. Clinical examination for the detection of protective sensation in the feet of diabetic patients. International Cooperative Group for Clinical Examination Research. J Gen Intern Med. 1999;14(7):418-424.

27. Davies M, Brophy S, Williams R, Taylor A. The prevalence, severity, and impact of painful diabetic peripheral neuropathy in type 2 diabetes. Diabetes Care. 2006;29(7):1518-1522.

28. Abbott CA, Malik RA, van Ross ER, Kulkarni J, Boulton AJ. Prevalence and characteristics of painful diabetic neuropathy in a large community-based diabetic population in the U.K. Diabetes Care. 2011;34(10):2220-2224.

29. Pregabalin and gabapentin: Red Book (2016). In Micromedex (Columbia Basin College Library ed; electronic version). Greenwood Village, CO: Truven Health Analytics. Available from: http://www.micromedexsolutions.com/. Accessed August 15, 2016.

30. Lesser H, Sharma U, LaMoreaux L, Poole RM. Pregabalin relieves symptoms of painful diabetic neuropathy: a randomized controlled trial. Neurology. 2004;63(11):2104-2110.

31. Moore RA, Wiffen PJ, Derry S, Toelle T, Rice AS. Gabapentin for chronic neuropathic pain and fibromyalgia in adults. Cochrane Database Syst Rev. 2014;4:CD007938.

32. McNicol ED, Midbari A, Eisenberg E. Opioids for neuropathic pain. Cochrane Database Syst Rev. 2013;8:CD006146.

33. Gimbel JS, Richards P, Portenoy RK. Controlled-release oxycodone for pain in diabetic neuropathy: a randomized controlled trial. Neurology. 2003;60(6):927-934.

34. Attal N, Cruccu G, Baron R, et al. EFNS guidelines on the pharmacological treatment of neuropathic pain: 2010 revision. Eur J Neurol. 2010;17(9):1113-e1188.

35. Moulin DE, Clark AJ, Gilron I, et al. Pharmacological management of chronic neuropathic pain - consensus statement and guidelines from the Canadian Pain Society. Pain Res Manag. 2007;12(1):13-21.

36. Rasu RS, Sohraby R, Cunningham L, Knell ME. Assessing chronic pain treatment practices and evaluating adherence to chronic pain clinical guidelines in outpatient practices in the United States. J Pain. 2013;14(6):568-578.

37. Sindrup SH, Otto M, Finnerup NB, Jensen TS. Antidepressants in the treatment of neuropathic pain. Basic Clin Pharmacol Toxicol. 2005;96(6):399-409.

38. Finnerup NB. Pharmacological management of neuropathic pain. Pain. 2010;18(9). Available from: http://iasp.files.cms-plus.com/ Content/ContentFolders/Publications2/PainClinicalUpdates/Archives/ PCU_18-9_final_1390260608342_7.pdf. Accessed February 1, 2017.

39. Rosenberg CJ, Watson JC. Treatment of painful diabetic peripheral neuropathy. Prosthet Orthot Int. 2015;39(1):17-28. 
The Journal of Pain Research is an international, peer reviewed, open access, online journal that welcomes laboratory and clinical findings in the fields of pain research and the prevention and management of pain. Original research, reviews, symposium reports, hypothesis formation and commentaries are all considered for publication
The manuscript management system is completely online and includes a very quick and fair peer-review system, which is all easy to use. Visit http://www.dovepress.com/testimonials.php to read real quotes from published authors. 